\title{
ELECTRIC FIELD OF A CHARGED WIRE AND A SLOTTED CYLINDRICAL CONDUCTOR
}

\author{
By Chester Snow
}

\begin{abstract}
The potential $U$, at any point $z=x+i y=r e^{i \theta}$, in the $x y$ plane is found which is due to a line charge $Q$ at the origin, perpendicular to the $x y$ plane, in the presence of a certain outer shield at zero potential. This shield consists of a cylindrical shell of no radial thickness having the line charge for its axis, whose trace on the $x y$ plane is that part of the circular arc $r=\alpha$ whose angle $\theta$ lies between the limits $-p$ and $p$ where $p$ is a positive angle less than $\pi$. This potential is given as the real part of the expression
\end{abstract}

$$
U+i V=Q \log \frac{(z-\alpha) \cos ^{2} \frac{p}{2}+(z+\alpha) \sin ^{2} \frac{p}{2}+\sqrt{\left(z-\alpha e^{i D}\right)\left(z-\alpha e^{-i D}\right)}}{-(z-\alpha) \cos ^{2} \frac{p}{2}+(z+\alpha) \sin ^{2} \frac{p}{2}+\sqrt{\left(z-\alpha e^{i D}\right)\left(z-\alpha e^{-i D}\right)}}
$$

where the radical denotes that branch whose real part is positive when the point $z$ is external to the circle. (This is a special case of the more general problem which is also solved in the paper, in which the line charge may have any position in the plane or may be absent entirely.)

The surface density $\sigma$ of the induced charge at a point $\theta$ on the charged arc is found from the above equation to have the value

$$
\sigma=-\frac{Q}{4 \pi \alpha}\left\{\frac{1}{\cos \frac{p}{2} \sqrt{\tan ^{2} \frac{p}{2}-\tan ^{2} \frac{\theta}{2}}} \pm 1\right\}
$$

the upper sign corresponding to the inner density (on the concave side), the lower to the outer. The outer density vanishes with the closing of the circle; that is, when $p=\pi$.

By making the transformation

$$
z=r e^{\mathrm{i} \theta}=Z^{\mathbf{n}}=R^{\mathbf{n}} e^{\mathbf{i n} \phi} \text { or }\left\{\begin{array}{l}
r=\mathrm{R}^{\mathbf{n}} \\
\theta=n \phi
\end{array}\right.
$$

the corresponding solution is obtained for $n$ equal, equally spaced concentric ares on the circle of radius $R=a=\alpha^{\frac{1}{n}}$ with a line charge at the origin.

\section{CONTENTS}

I. Introduction

II. Potential of a line source surrounded by concentric circular cylindrical arcs at zero potential. 


\section{INTRODUCTION}

The electrostatic problem here solved is that of finding the potential due to a charged conducting wire (treated as a line charge) which is surrounded by a slotted cylinder at zero potential. This outer cylinder is of negligible thickness radially and the slots are parallel to its generating lines, equal, and equally spaced. This is an idealized case, which was undertaken for the purpose of estimating the effect of perforating an outer cylindrical shield upon the strength of field. ${ }^{1}$ The problem thus simplified admits of an exact solution in finite terms and the methods used possess a mathematical interest. The results may also be of practical value in other distinct lines of physical research.

The mathematical problem of discovering a potential function which is a solution of Laplace's equation, and which reduces to certain assigned constant values on the conducting contours which bound the space, is in many cases simplified by a succession of transformations between complex variables, each of which may be represented on a plane. For, as is well known, the real part, and the imaginary part, of a complex function is each a solution of Laplace's equation in the particular variables used, so that one may be assured that if a potential function which fits the required boundary values is arrived at in this manner, then the partial differential equation will also be satisfied. With the mind thus freed of any responsibility for the differential equation, the whole attention may then be devoted to devising or discovering transformations from plane to plane in which the conducting boundary curves are more amenable to the known processes of building up a solution of the potential equation which shall take on the assigned values upon them. If this can be done for any complex variable, it is then an easy matter to retrace the steps of the transformation and obtain the potential function which was originally desired. It is frequently found that these intentional distortions of the space rob the original problem of much of its complexity, and in many cases lead to finite solutions of problems which for direct attack would seem to be very formidable.

\section{POTENTIAL OF A LINE SOURCE SURROUNDED BY CON- CENTRIC CIRCULAR CYLINDRICAL ARCS AT ZERO POTENTIAL}

If the potential is found for the case where there is only one circular arc, whose angular range is from $-p$ to $p$ where $0<p<\pi$, for the $z$ plane, then the original problem of $n$ arcs may be readily found from this by the transformation $Z^{n}=z$. Postponing this transfor-

\footnotetext{
1 The question arises in the use of the corona voltmeter as used by Professor Whitehead. The outer cylinder is perforated to permit the passage through it of ions on their way to an outer collecting electrode.
} 
mation for the present, it will be more simple to begin with the transformation

$$
x+i y=z=\frac{\alpha\left(i-z^{\prime}\right)}{i+z^{\prime}}
$$

that is

$$
x=\frac{\alpha\left(1-x^{\prime 2}-y^{\prime 2}\right)}{x^{\prime 2}+\left(y^{\prime}+1\right)^{2}} \quad y=\frac{2 \alpha x^{\prime}}{x^{\prime 2}+\left(y^{\prime}+1\right)^{2}}
$$

This may also be written

which is the same as

$$
x^{\prime}+i y^{\prime}=z^{\prime}=i \frac{\alpha-z}{\alpha+z}
$$

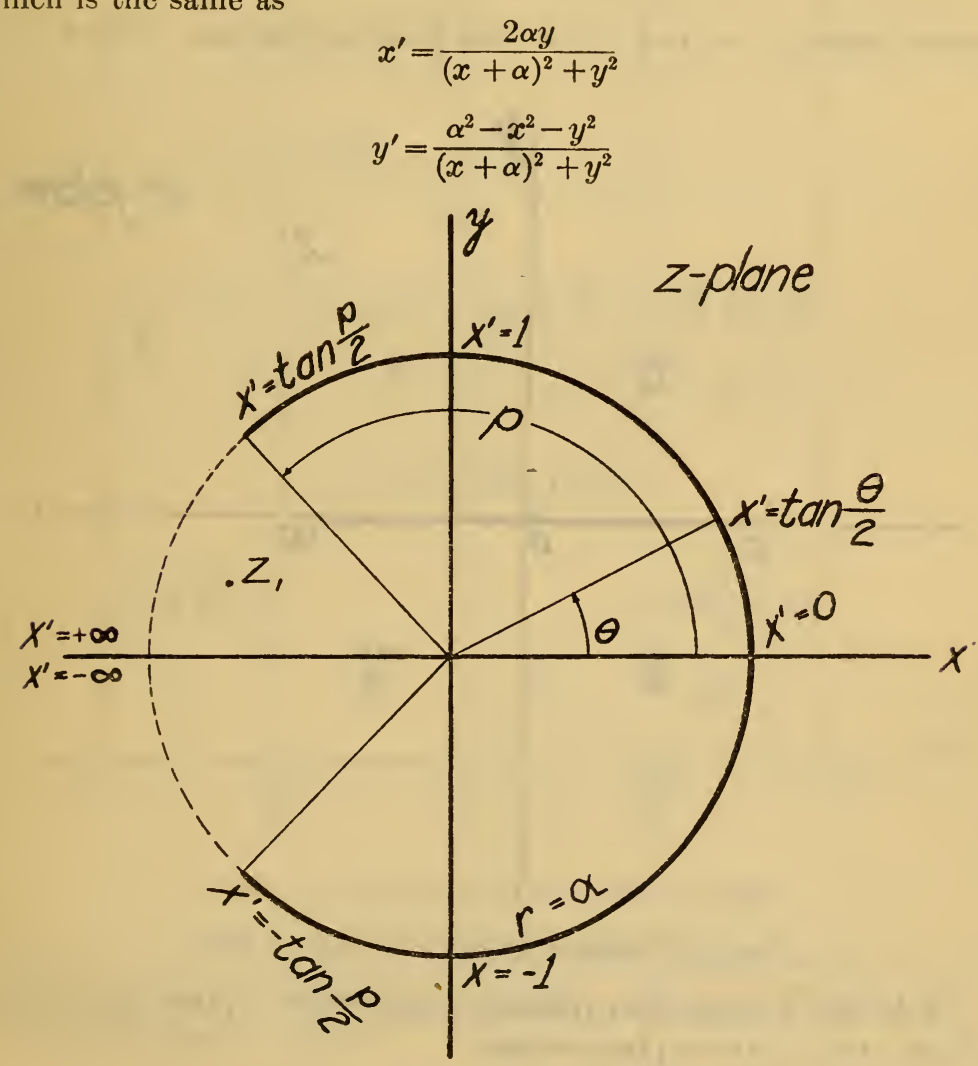

FIG. 1.-Section of charged cylinder in z plane

This gives a one-to-one correspondence between the $z$ and $z^{\prime}$ planes. The circle of radius $\alpha$ and center at the origin in the $z$ plane corresponds to the entire real axis $y^{\prime}=0$ in the $z^{\prime}$ plane. Points inside this circle correspond to points in the upper half of the $z^{\prime}$ plane. A certain part of this circular arc will be assumed to be the trace of a cylindrical conductor at zero potential. 
In Figure 1 the circle $r=\alpha$ is shown in the $z$ plane and the values of $x^{\prime}$, which correspond to several points on this arc. The conducting arc is given by $r=\alpha,-p<\theta<p$.

In order to find a real potential function $U(x y)$ produced at a point $z$ in the $z$ plane by a line source of strength $Q$ at some point $z_{1}=x_{1}+i y_{1}$, in the $z$ plane, in the presence of the circular cylindrical arc at zero potential, we must find a potential $U\left(x^{\prime} y^{\prime}\right)$ for the $z^{\prime}$ plane which corresponds to a line source at the point $z^{\prime}{ }_{1}=x^{\prime}{ }_{1}+i y_{1}{ }_{1}$ $=i\left(\frac{\alpha-z_{1}}{\alpha+z_{1}}\right)$ in the presence of a strip at zero potential, which strip extends from $x^{\prime}=-c$ to $x^{\prime}=+c=\tan \frac{p}{2}$ on the real axis. (See fig. 2.)

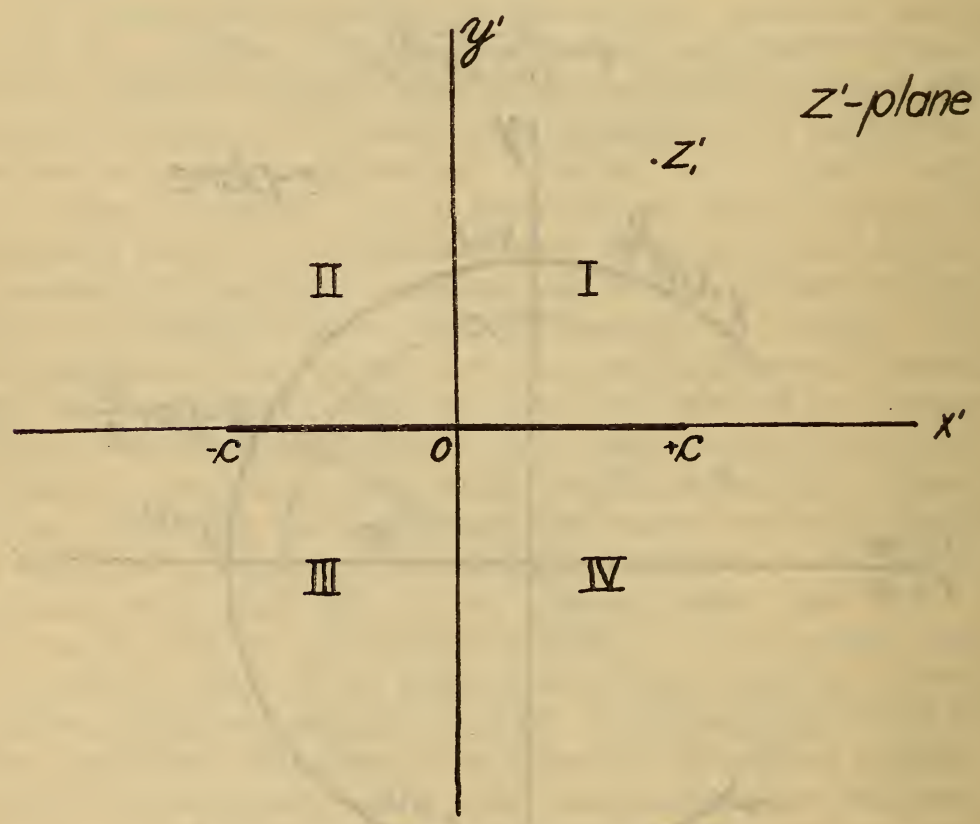

Fig. 2.-Section of charged strip in the $z^{\prime}$ plane

To do this we may next transform this entire $z^{\prime}$ plane into a semiinfinite strip on the $w$ plane where

$$
w \equiv u+i v\left\{\begin{array}{r}
-\pi \leqq u \leqq \pi \\
0 \leqq v \leqq \infty
\end{array}\right\} c=\tan \frac{p}{2}
$$

by the equation

$$
z^{\prime}=c \cos w \text { or }\left\{\begin{array}{l}
x^{\prime}=c \cos u \cosh v \\
y^{\prime}=-c \sin u \sinh v
\end{array}\right\}
$$


The straight line $v=v_{0}>0$ where $-\pi \leqq u \leqq \pi$ corresponds to an ellipse in the $z^{\prime}$ plane whose foci are at $x^{\prime}= \pm c, y^{\prime}=0$. In particular, the line $v=0(-\pi<u<\pi)$ corresponds to the conductor at zero potential in the $z^{\prime}$ plane. Some point $w_{1}=u_{1}+i v_{1}$ where $z_{1}^{\prime}=c$ $\cos w_{1}$ corresponds to the line charge. One must then find a potential $U(u v)$ for the $w$ plane corresponding to the line charge $Q$ at the

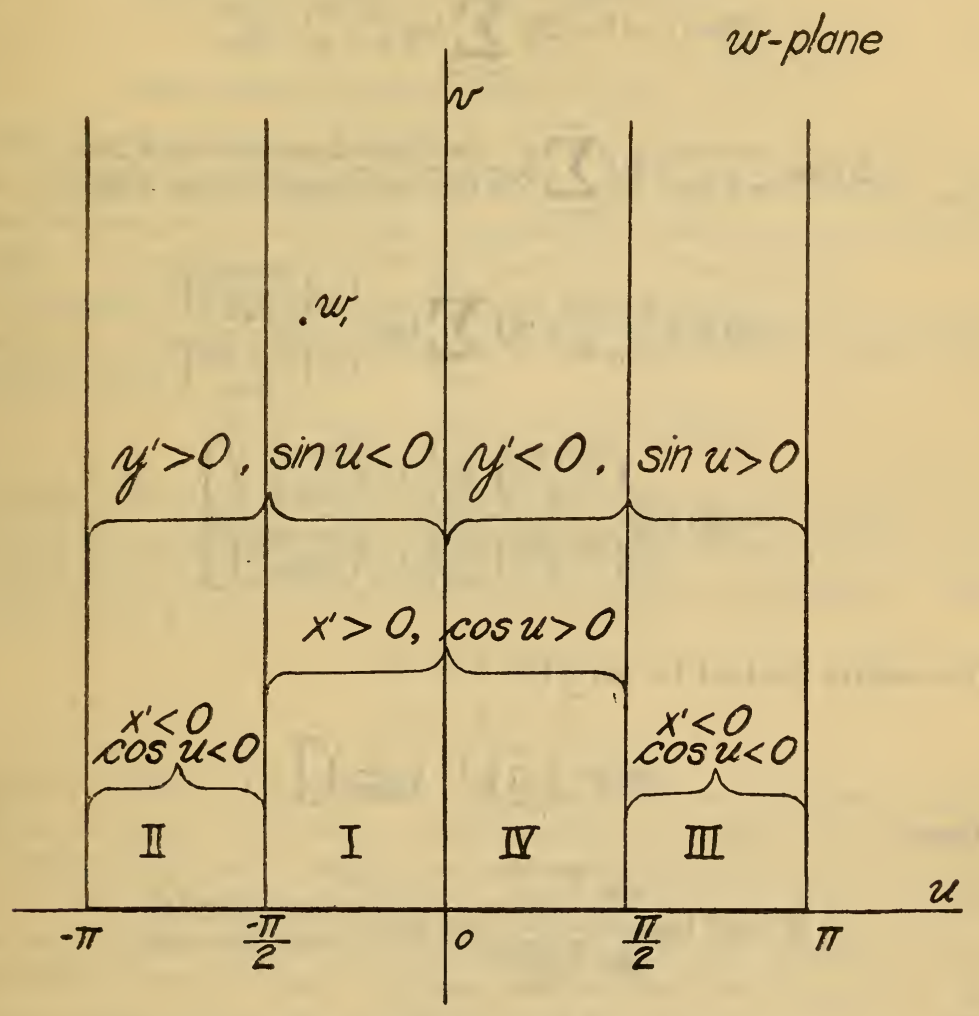

Fig. 3.-Semi-infinite strip in the $w$ plane

point $w_{1}$ in this strip of the $w$ plane, which vanishes when $v=0$, and which must be periodic in $u$ of period $2 \pi$. Now it is evident that the real part of the function

$$
2 Q \log \frac{w-\bar{w}_{1}}{w-w_{1}}\left(=2 Q \log \left[\frac{w-\left(u_{1}-i v_{1}\right)}{w-\left(u_{1}+i v_{1}\right)}\right]\right)
$$

will vanish on the entire real axis of $w$ and will contain the required singularity corresponding to the line charge in the upper strip, namely, $-2 Q \log \left(w-w_{1}\right)$. The term

$$
2 Q \log \left(w-\bar{w}_{1}\right)=-2(-Q) \log \left[w-\left(u_{1}-i v_{1}\right)\right]
$$


corresponds to a negative line charge $-Q$ at its image point (or conjugate point) an equal distance below the real axis. This, however, is not a periodic function of $u$. Such a periodic solution may be obtained by imagining such pairs of equal and opposite line charges at conjugate points to be repeated periodically in $u$ with period $2 \pi$, so that

$$
\begin{gathered}
W \equiv U+i V=2 Q \sum_{n=-\infty}^{\infty} \log \frac{w-\bar{w}_{1}-2 \pi n}{w-w_{1}-2 \pi n} \\
=2 Q \log \frac{w-\bar{w}_{1}}{w-w_{1}}+2 Q \sum_{n=1}^{\infty} \log \frac{\left(w-\bar{w}_{1}-2 \pi n\right)\left(w-\bar{w}_{1}+2 \pi n\right)}{\left(w-w_{1}-2 \pi n\right)\left(w-w_{1}+2 \pi n\right)} \\
=2 Q \log \frac{w-\bar{w}_{1}}{w-w_{1}}+2 Q \sum_{n=1}^{\infty} \log \frac{1-\left(\frac{w-\bar{w}_{1}}{2 \pi n}\right)^{2}}{1-\left(\frac{w-w_{1}}{2 \pi n}\right)^{2}} \\
=2 Q \log \frac{\left(\frac{w-\bar{w}_{1}}{2}\right) \prod_{n=1}^{\infty}\left[1-\left(\frac{w-\bar{w}_{1}}{2 \pi n}\right)^{2}\right]}{\left(\frac{w-w_{1}}{2}\right) \prod_{n=1}^{\infty}\left[1-\left(\frac{w-w_{1}}{2 \pi n}\right)^{2}\right]}
\end{gathered}
$$

The infinite product for $\sin \frac{z}{2}$ is

Hence

$$
\sin \frac{z}{2}=\frac{z}{2} \prod_{n=1}^{\infty}\left[1-\left(\frac{z}{2 \pi n}\right)^{2}\right]
$$

$$
W=2 Q \log \frac{\sin \frac{w-\bar{w}_{1}}{2}}{\sin \frac{w-w_{1}}{2}}=Q \log \frac{1-\cos \left(w-\bar{w}_{1}\right)}{1-\cos \left(w-w_{1}\right)}
$$

Or

$$
W \equiv U+i V=Q \log \frac{1-\cos \bar{w}_{1} \cos w-\sin \bar{w}_{1} \sin w}{1-\cos w_{1} \cos w-\sin w_{1} \sin w}
$$

It is evident, therefore, that the real part of the second member of equation (3) gives a potential function $U$ which is periodic in $u$ of period $2 \pi$ and which vanishes at the base of the semiinfinite strip in the $w$ plane, and has one singularity in this strip corresponding to the line charge at $w_{1}$.

If we now return to the $z^{\prime}$ plane, replacing $w$ in terms of $z^{\prime}$ in equation (3), this will give the Green's function $U$ for the $z^{\prime}$ plane external to that part of the real axis between $-c$ and $c$, for $U$ will vanish here and will have a logarithmic singularity at the one point 
$z^{\prime}{ }_{1}$ in the $z^{\prime}$ plane corresponding to the one singularity at $w_{1}$ in the half strip.

If $\bar{z}_{1}{ }^{\prime}$ is the conjugate of $z_{1}{ }^{\prime}$ we may place

$$
\begin{aligned}
& \cos w=\frac{z^{\prime}}{c}, \cos w_{1}=\frac{z_{1}^{\prime}}{c}, \cos \bar{w}_{1}=\frac{\bar{z}_{1}^{\prime}}{c}, \\
& \sin w=\sin u \cosh v+i \cos u \sinh v=\frac{\sqrt{c^{2}-z^{\prime 2}}}{c} \\
& \sin w_{1}=\sin u_{1} \cosh v_{1}+i \cos u_{1} \sinh v_{1}=\frac{\sqrt{c^{2}-z_{1}^{\prime}}}{c}
\end{aligned}
$$

where, by the radical $\sqrt{c^{2}-z^{\prime 2}}$ we must understand that branch of the double-valued function of $z^{\prime}$, whose real part has the same algebraic $\operatorname{sign}$ as $\sin u$ and whose imaginary part has the same algebraic sign as $\cos u$ (because $v$ is always positive). (Sec fig. 3.)

Similarly $\sqrt{c^{2}-{z^{\prime}}_{1}^{2}}$ must denote that branch whose real and imaginary parts have the algebraic signs of $\sin u_{1}$ and $\cos u_{1}$, respectively. Finally, since

$$
\sin \bar{w}_{1}=\sin u_{1} \cosh v_{1}-i \cos u_{1} \sinh v_{1}
$$

we may replace $\sin \bar{w}_{1}$ by $\overline{\sqrt{c^{2}-z^{\prime 2}{ }^{2}}}$; that is, the conjugate of $\sqrt{c^{2}-{z^{\prime}{ }_{1}^{2}}^{2}}$. This interpretation of the branch of the square root $\sqrt{c^{2}-z^{\prime 2}}$ may also be put in terms of the position of the point $z^{\prime}$ in the plane. Thus if $\sqrt{c^{2}-z^{\prime 2}}=A+i B$

$$
\begin{aligned}
& A>0 \text { if } y^{\prime}<0 \\
& A<0 \text { if } y^{\prime}>0
\end{aligned}
$$

Stated in words: The complex radical $\sqrt{c^{2}-z^{\prime 2}}$ denotes that branch whose real part $A$ is positive when the point $z^{\prime}$ lies below the real axis and whose real part is negative when the point $z^{\prime}$ lies above the real axis. This real part will vanish when $z^{\prime}$ lies on the real axis outside the conducting strip, but will approach equal and opposite values different from zero when $z^{\prime}$ approaches this strip from opposite sides. The imaginary part, $B$, of $\sqrt{c^{2}-z^{\prime 2}}$ will have the same sign as $x^{\prime}$, being positive, in general, if $z^{\prime}$ lies to the right of the $y^{\prime}$ axis and negative if to the left. But it will vanish on the conducting strip.

Similarly by $\sqrt{c^{2}-z_{1}^{\prime}}$ must be understood that branch which depends upon the position of the point $z_{1}^{\prime}$ in precisely the same manner as $\sqrt{c^{2}-z^{\prime 2}}$ depends upon $z^{\prime}$. With this understanding, equation (3) becomes

$$
W=U+i V=Q \log \frac{c^{2}-\bar{z}^{\prime}{ }_{1} z^{\prime}-\sqrt{c^{2}-z^{\prime}{ }_{1}^{2}} \sqrt{c^{2}-z^{\prime 2}}}{c^{2}-z^{\prime}{ }_{1} z^{\prime}-\sqrt{c^{2}-z^{\prime}{ }_{1}^{2}} \sqrt{c^{2}-z^{\prime 2}}}
$$

The real part of this vanishes when the point $z^{\prime}$ comes on the conducting strip, for then $z^{\prime}$ is real and numerically less than $c$. Hence, $6849^{\circ}-26-2$ 
whenever this strip is approached either from above or below, the numerator and denominator of this fraction become conjugate imaginaries and the real part of the logarithm vanishes.

It might appear, at first glance, that the second member of equation (4) would vanish if $c$ were allowed to become infinite, whatever the position of the points $z_{1}^{\prime}$ and $z^{\prime}$. This is not the case, however, for

$$
W=Q \log \frac{1-\sqrt{1-\frac{z_{1}^{\prime 2}}{c^{2}}} \sqrt{1-\frac{z^{\prime 2}}{c^{2}}}-\left(\frac{z_{1}^{\prime}}{c}\right)\left(\frac{z^{\prime}}{c}\right)}{1-\sqrt{1-\frac{z_{1}^{\prime}}{c^{2}}} \sqrt{1-\frac{z^{\prime 2}}{c^{2}}}-\left(\frac{z_{1}^{\prime}}{c}\right)\left(\frac{z^{\prime}}{c}\right)}
$$

where

$$
\begin{aligned}
\sqrt{1-\frac{z^{\prime 2}}{c^{2}}} & =1-\frac{z^{\prime 2}}{2 c^{2}} \ldots \ldots \text { if } z^{\prime} \text { is below the real axis } \\
& =-1+\frac{z^{\prime 2}}{2 c^{2}} \ldots \text { if } z^{\prime} \text { is above the real axis }
\end{aligned}
$$

Similarly

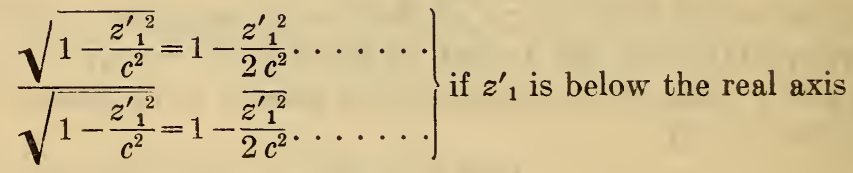

$$
\begin{aligned}
& \left.\frac{\sqrt{1-\frac{z_{1}^{\prime}{ }^{2}}{c^{2}}}}{\sqrt{1-\frac{z_{1}^{\prime}}{c^{2}}}}=-1+\frac{\frac{z_{1}^{\prime}{ }_{1}^{2}}{2 c^{2}} \ldots}{\frac{z_{1}^{\prime}}{2 c^{2}}} \ldots \ldots .\right) \text { if } z_{1}^{\prime} \text { is above the real axis }
\end{aligned}
$$

Hence, if both $z^{\prime}$ and $z^{\prime}{ }_{1}$ are above the real axis or both below it

$$
W=2 Q \log \frac{z^{\prime}-\overline{z_{1}^{\prime}}}{z^{\prime}-z_{1}^{\prime}}
$$

But if $z^{\prime}$ and $z_{1}^{\prime}$ are on opposite sides of the real axis then $W=0$. Thus the expression equation (4) reduces to the ordinary one when the conductor at zero potential extends along the entire real axis.

To return finally to the original $z$ plane, we may, in equation (4), replace $z^{\prime}$ by

$$
\begin{gathered}
-i\left(\frac{z-\alpha}{z+\alpha}\right), c \text { by } \tan \frac{p}{2}, z_{1}^{\prime} \text { by }-i\left(\frac{z_{1}-\alpha}{z_{1}+\alpha}\right), \text { and } \bar{z}^{\prime}{ }_{1} \text { by }\left[-i\left(\frac{z_{1}-\alpha}{z_{1}+\alpha}\right)\right] \\
=+i\left(\frac{\bar{z}_{1}-\alpha}{\bar{z}_{1}+\alpha}\right) .
\end{gathered}
$$


This gives

$$
\begin{aligned}
W=U+i V=Q \log & {\left[\frac{\tan ^{2} \frac{p}{2}-\left(\frac{\bar{z}_{1}-\alpha}{z_{1}+\alpha}\right)\left(\frac{z-\alpha}{z+\alpha}\right)}{\left.-\sqrt{\tan ^{2} \frac{p}{2}+\left(\frac{z_{1}-\alpha}{z_{1}+\alpha}\right)^{2}} \sqrt{\tan ^{2} \frac{p}{2}+\left(\frac{z-\alpha}{z+\alpha}\right)^{2}}\right]}\right.} \\
-Q \log [ & {\left[\frac{\tan ^{2} \frac{p}{2}+\left(\frac{z_{1}-\alpha}{z_{1}+\alpha}\right)\left(\frac{z-\alpha}{z+\alpha}\right)}{}\right.} \\
& \left.-\sqrt{\tan ^{2} \frac{p}{2}+\left(\frac{z_{1}-\alpha}{z_{1}+\alpha}\right)^{2}} \sqrt{\tan ^{2} \frac{p}{2}+\left(\frac{z-\alpha}{z+\alpha}\right)^{2}}\right]
\end{aligned}
$$

where if

$$
\begin{aligned}
& \sqrt{\tan ^{2} \frac{p}{2}+\left(\frac{z-\alpha}{z+\alpha}\right)^{2}} \equiv A+i B \\
& \sqrt{\tan ^{2} \frac{p}{2}+\left(\frac{z_{1}-\alpha}{z_{1}+\alpha}\right)^{2}} \equiv A_{1}+i B_{1} \\
& \sqrt{\tan ^{2} \frac{p}{2}+\left(\frac{z_{1}-\alpha}{z_{1}+\alpha}\right)^{2}} \equiv A_{1}-i B_{1}
\end{aligned}
$$

then $A$ (or $A_{1}$ ) is positive or negative according as $z$ (or $z_{1}$ ) is outside or inside the circle $z=\alpha e^{1 \theta} ; B$ (or $B_{1}$ ) is positive or negative according as $z$ (or $z_{1}$ ) is above or below the real axis. If $z$ (or $z_{1}$ ) is on that part of the circle which is outside the conducting arc, then $A$ (or $A_{1}$ ) vanishes. If $z$ (or $z_{1}$ ) approaches the conducting part of the circular arc, then $B$ (or $B_{1}$ ) vanishes while $A= \pm \sqrt{\tan ^{2} \frac{p}{2}-\tan ^{2} \frac{\theta}{2}}$, (or $A_{1}=$ $\pm \sqrt{\left.\tan ^{2} \frac{p}{2}-\tan ^{2} \frac{\theta_{1}}{2}\right)}$ being positive or negative according to whether the conducting arc is approached from the outside or the inside of the circle. Since $\frac{z-\alpha}{z+\alpha}$ becomes $i \tan \frac{\theta}{2}$ then for either direction of approach the numerator and denominator of the fraction in the second member of equation (5) become conjugate imaginaries so that the real part of the logarithm (and hence $U$ ) vanishes. From the mode of derivation equation (5) it is evident that the only singularity of the function $W$ in the entire $z$ plane external to the conducting arc is at the point $z=z_{1}$. The two extremities of the conducting arc at $z=\alpha e^{1 \mathrm{p}}$ and $z=\alpha e^{-\mathrm{tp}}$ are readily seen to be branch points, for we may write

$$
\sqrt{\tan ^{2} \frac{p}{2}+\left(\frac{z-\alpha}{z+\alpha}\right)^{2}}=\sqrt{\frac{\left(z-\alpha e^{1 \mathrm{p}}\right)\left(z-\alpha e^{-1 \mathrm{p}}\right)}{(z+\alpha)^{2} \cos ^{2} \frac{p}{2}}}
$$

If one imagines the conducting arc to increase until its extremities touch, thus closing the circle so that $p=\pi$, this corresponds to the case where $c=\tan \frac{p}{2}=\infty$; that is, when the entire real axis of $z^{\prime}$ is 
at zero potential. The same argument which was applied to this particular case in the $z^{\prime}$ plane, may be applied here, and it shows that if one of the two points $z$ and $z_{1}$ is within and the other without the circle the value of $W$ is zero; but if both points are within or both without the circle, the value of $W$ reduces to the familiar one for the case of a line charge in the presence of a complete circular boundary at zero potential.

If as a particular case the line charge is on the real axis, then $z_{1}$ is real $\left(=x_{1}\right)$. In this case the dashes may be omitted in equation (5) since they serve to indicate the conjugate of quantities which now are real. Equation (5) then becomes

$$
\begin{array}{r}
{\left[\tan ^{2} \frac{p}{2}-\frac{x_{1}-\alpha}{x_{1}+\alpha} \sqrt{1+\left(\frac{x_{1}+\alpha}{x_{1}-\alpha}\right)^{2} \tan ^{2} \frac{p}{2}} \sqrt{\tan ^{2} \frac{p}{2}+\left(\frac{z-\alpha}{z+\alpha}\right)^{2}}\right.} \\
\left.-\left(\frac{x_{1}-\alpha}{x_{1}+\alpha}\right)\left(\frac{z-\alpha}{z+\alpha}\right)\right] \\
{\left[\begin{array}{l}
\tan ^{2} \frac{p}{2}-\frac{x_{1}-\alpha}{x_{1}+\alpha} \sqrt{1+\left(\frac{x_{1}+\alpha}{x_{1}-\alpha}\right)^{2} \tan ^{2} \frac{p}{2}} \sqrt{\tan ^{2} \frac{p}{2}+\left(\frac{z-\alpha}{z+\alpha}\right)^{2}} \\
\left.+\left(\frac{x_{1}-\alpha}{x_{1}+\alpha}\right)\left(\frac{z-\alpha}{z+\alpha}\right)\right]
\end{array}\right.}
\end{array}
$$

where the radical involving $x_{1}$ is to be taken as a positive real.

In case the line charge is at the origin (center of the circle) then $x_{1}=0$ and equation (6) becomes

$$
\begin{gathered}
W=U+i V=Q \log \frac{\sin ^{2} \frac{p}{2}+\sqrt{\frac{\left(z-\alpha e^{1 \mathrm{p}}\right)\left(z-\alpha e^{-1 \mathrm{p}}\right)}{(z+\alpha)^{2}}}+\frac{z-\alpha}{z+\alpha} \cos ^{2} \frac{p}{2}}{\sin ^{2} \frac{p}{2}+\sqrt{\frac{\left(z-\alpha e^{\mathrm{Ip}}\right)\left(z-\alpha e^{-1 \mathrm{p}}\right)}{(z+\alpha)^{2}}}-\frac{z-\alpha}{z+\alpha} \cos ^{2} \frac{p}{2}} \\
{\left[(z-\alpha) \cos ^{2} \frac{p}{2}+(z+\alpha) \sin ^{2} \frac{p}{2}\right.} \\
=Q \log \frac{\left.+\sqrt{\left(z-\alpha e^{\mathrm{p}}\right)\left(z-\alpha e^{-1 \mathrm{p}}\right)}\right]}{\left[-(z-\alpha) \cos ^{2} \frac{p}{2}+(z-\alpha) \sin ^{2} \frac{p}{2}\right.} \\
\left.+\sqrt{\left(z-\alpha e^{1 \mathrm{p}}\right)\left(z-\alpha e^{-\mathrm{lp}}\right)}\right] \\
=Q \log \frac{z_{1} \cos ^{2} \frac{p}{2}+z_{3} \sin ^{2} \frac{p}{2}+\sqrt{z_{2} z_{4}}}{-z_{1} \cos ^{2} \frac{p}{2}+z_{3} \sin ^{2} \frac{p}{2}+\sqrt{z_{2} z_{4}}}
\end{gathered}
$$

where the four vectors $z_{1}, z_{2}, z_{3}$, and $z_{4}$ are drawn to the variable point $z=r e^{i \theta}$ from the four fixed points $\alpha, \alpha e^{i p},-\alpha, \alpha e^{-i p}$, respectively, so that (fig. 4)

$$
\begin{array}{ll}
z_{1} \equiv z-\alpha=r_{1} e^{i \theta_{1}} & z_{3} \equiv z+\alpha=r_{3} e^{i \theta_{3}} \\
z_{2} \equiv z-\alpha e^{i p}=r_{2} e^{i \theta_{2}} & z_{4} \equiv z-\alpha e-i p=r_{4} e^{i \theta_{4}}
\end{array}
$$


It must be remembered that the radicals in equation (7) designate that branch whose real part is positive when the point $z$ is outside the circle $r=\alpha$. The ambiguity may be removed by making the following specifications as to the angles $\theta_{1}, \theta_{2}, \theta_{3}, \theta_{4}$. The value to be assigned to each of these angles at any point $z$ in the plane, is any value which it may attain by continuous variation as the point is reached after starting from an infinite distance to the right on the real axis (where each of these angles has the value zero), provided that the point $z$ never crosses that part of the circular arc which is at zero potential. The four vectors with their moduli and angles are shown in Figure 4. With this understanding equation (7) may be written

$$
W=U+i V=Q \log \frac{r_{1} e^{\mathrm{i} \theta_{1}} \cos ^{2} \frac{p}{2}+r_{3} e^{\mathrm{i} \theta_{4}} \sin ^{2} \frac{p}{2}+\sqrt{r_{2} r_{4}} e^{\mathrm{i} \frac{\theta_{3}+\theta_{4}}{2}}}{-r_{1} e^{\mathrm{i} \theta_{4}} \cos ^{2} \frac{p}{2}+r_{3} e^{\mathrm{i} \theta_{2}} \sin ^{2} \frac{p}{2}+\sqrt{r_{2} r_{4}} e^{\mathrm{i} \frac{\theta_{3}+\theta_{4}}{2}}}
$$

The equations (7) or (9) then define a potential function $U$ which is continuous and single valued at all points external to the charged arc except the origin which contains the line charge of strength $Q$. This potential will vanish on the charged arc. The corresponding flux function $V$ which is conjugate to $U$ will approach different values on opposite sides of the same point on the conducting arc.

To verify these statements we may note first that when $\frac{z}{\alpha}$ is small equation (7) gives the following development

$$
\begin{aligned}
W= & Q \log \frac{\alpha^{2}}{z^{2} \sin ^{2} p} \\
& +Q \log \left[\cos p-\frac{z}{\alpha}-\sqrt{\left(1-\frac{z e^{\mathrm{ip}}}{\alpha}\right)\left(1-\frac{z e^{-\mathrm{ip}}}{\alpha}\right)}\right] \\
& +Q \log \left[1-\frac{z \cos p}{\alpha}-\sqrt{\left(1-\frac{z e^{\mathrm{ip}}}{\alpha}\right)\left(1-\frac{z e^{-\mathrm{ip}}}{\alpha}\right)}\right] \\
= & \left.2 Q \mid-\log z+\log \frac{\alpha}{\sin \frac{p}{2}}-\cos ^{2} \frac{p}{2} \cdot \frac{z}{\alpha}+\cos ^{2} \frac{p}{2}\left(1-\frac{3}{2} \cos ^{2} \frac{p}{2}\right) \frac{z^{2}}{\alpha^{2}}+\cdots\right\}
\end{aligned}
$$

This shows that the principal part of $W$ when $\frac{z}{\alpha}$ is small is $-2 Q \log z$ which corresponds to the line charge of strength $Q$ at the origin. 
To show that the potential $U$ vanishes when the point $z$ approaches the charged arc, we note that if $z$ is any point on the entire circular arc, then $z=\alpha e^{i \theta}, \frac{z-\alpha}{z+\alpha}=i \tan \frac{\theta}{2}$ and

$$
\sqrt{\frac{\left(z-\alpha e^{1 p}\right)\left(z-\alpha e^{-1 p}\right)}{(z+\alpha)^{2}}}=\frac{\sqrt{\sin ^{2} \frac{p}{2}-\sin ^{2} \frac{\theta}{2}}}{\cos \frac{\theta}{2}}
$$

so that the potential $U$ at any point on the circle $r=\alpha$ is the real part of

$$
W=Q \log \frac{\sin ^{2} \frac{p}{2} \cos \frac{\theta}{2}+\sqrt{\sin ^{2} \frac{p}{2}-\sin ^{2} \frac{\theta}{2}}+i \sin \frac{\theta}{2} \cos ^{2} \frac{p}{2}}{\sin ^{2} \frac{p}{2} \cos \frac{\theta}{2}+\sqrt{\sin ^{2} \frac{p}{2}-\sin ^{2} \frac{\theta}{2}}-i \sin \frac{\theta}{2} \cos ^{2} \frac{p}{2}}
$$

If, further, the point is on that part of the circle which is conducting $-p<\theta<p$ the radical in equation (11) is real, the numerator and denominator are conjugate imaginaries, and the real part of the logarithm, the potential, vanishes. If, on the other hand, the point is on that part of the circular arc not occupied by the conductor, the radical is imaginary and the potential by equation (11) has the variable value

$$
\begin{aligned}
U=2 Q \log \left[\left(\frac{\sin \frac{\theta}{2}}{\sin \frac{p}{2}}\right)^{2}+\sqrt{\left(\frac{\sin \frac{\theta}{2}}{\sin \frac{p}{2}}\right)^{2}-1}\right] \\
=2 Q \cosh ^{-1}\left(\frac{\sin \frac{\theta}{2}}{\sin \frac{p}{2}}\right)
\end{aligned}
$$

The flux function $V$ is also of interest for its level lines are the lines of force, and its value at the conducting surface measures the surface density of charge there. To find $V_{0}$ at any point $\theta$ on the outside of the conducting arc, suppose that the point $P$ of Figure 4 comes up to such a point from the outside. The point is given by $z=(\alpha+0) e^{\mathrm{i} \theta}$. From the geometry of the circle if $\theta>0$

$$
\left.\begin{array}{rlrl}
\sqrt{r_{2} r_{4}} & =2 \alpha \sqrt{\sin ^{2} \frac{p}{2}-\sin ^{2} \frac{\theta}{2}} \text { and } \frac{\theta_{2}+\theta_{4}}{2} & =\frac{\theta}{2} \\
r_{1} & =2 \alpha \sin \frac{\theta}{2} & \theta_{1} & =\frac{\theta}{2}+\frac{\pi}{2} \\
r_{3} & =2 \alpha \cos \frac{\theta}{2} & \theta_{3} & =\frac{\theta}{2}
\end{array}\right\}
$$


Substituting these values in equation (9) gives since $U=0$

$$
V_{0}=\frac{Q}{i} \log \frac{\cos \frac{\theta}{2} \sin ^{2} \frac{p}{2}+\sqrt{\sin ^{2} \frac{p}{2}-\sin ^{2} \frac{\theta}{2}}+i \sin \frac{\theta}{2} \cos ^{\frac{1}{2}} \frac{p}{2}}{\cos \frac{\theta}{2} \sin ^{2} \frac{p}{2}+\sqrt{\sin ^{2} \frac{p}{2}-\sin ^{2} \frac{\theta}{2}-i \sin \frac{\theta}{2} \cos ^{2} \frac{p}{2}}}
$$$$
=Q\left[2 \sin ^{-1}\left(\frac{\sin \frac{\theta}{2}}{\sin \frac{p}{2}}\right)-\theta\right]
$$

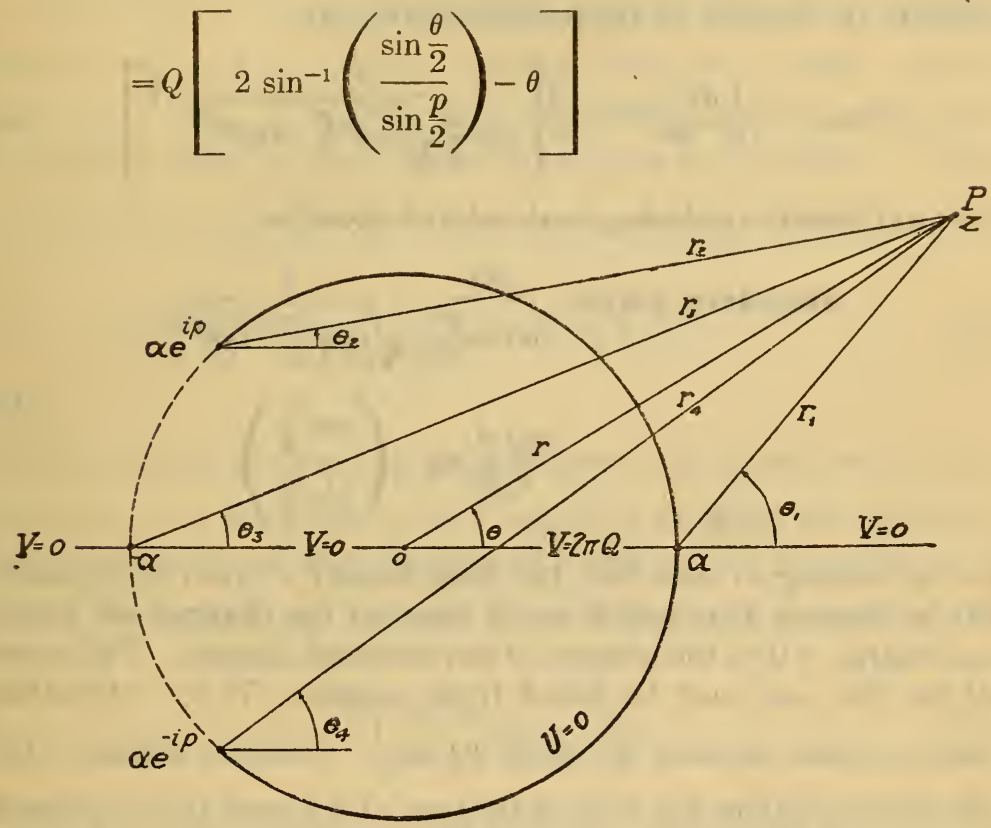

FIG. 4.-Polar coordinates in the $z$ plane

If the point $P$ now moves up around the upper extremity of the charged arc and comes back to this same part $\theta$ of the charged are from the inside, $\theta_{2}$ is increased by $2 \pi$ and all the other angles and moduli in equation (13) return to their original values. This gives for the value of $V_{i}$ on the inner side of the same point, by equation (9)

$$
\begin{aligned}
V_{i} & =\frac{Q}{i} \log \frac{\cos \frac{\theta}{2} \sin ^{2} \frac{p}{2}-\sqrt{\sin ^{2} \frac{p}{2}-\sin ^{2} \frac{\theta}{2}}+i \sin \frac{\theta}{2} \cos ^{2} \frac{p}{2}}{\cos \frac{\theta}{2} \sin ^{2} \frac{p}{2}-\sqrt{\sin ^{2} \frac{p}{2}-\sin ^{2} \frac{\theta}{2}}-i \sin \frac{\theta}{2} \cos ^{2} \frac{p}{2}} \\
& =Q\left[2 \pi-\sin ^{-1}\left(\frac{\sin \frac{\theta}{2}}{\sin \frac{p}{2}}\right)-\theta\right]
\end{aligned}
$$


By means of equations (14) and (15) we may find the surface density $\sigma$ of the charge on either side of the conducting arc. The density $\sigma_{1}$ on the inside is given by

$$
4 \pi \sigma_{i}=\left(\frac{\partial U}{\partial r}\right)_{\mathrm{r}=\alpha-0}=\frac{1}{\alpha} \frac{\partial V_{\alpha-0}}{\partial \theta}=-\frac{Q}{\alpha}\left[\frac{1}{\cos \frac{p}{2} \sqrt{\tan ^{2} \frac{p}{2}-\tan ^{2} \frac{\theta}{2}}}+1\right]
$$

Similarly the density on the outside is given by

$$
4 \pi \sigma_{0}=-\frac{1}{\alpha} \frac{\partial V_{\alpha+0}}{\partial \theta}=-\frac{Q}{\alpha}\left[\frac{1}{\cos \frac{p}{2} \sqrt{\tan ^{2} \frac{p}{2}-\tan ^{2} \frac{\theta}{2}}}-1\right]
$$

The total density (including both sides) is given by

$$
\begin{aligned}
4 \pi \sigma=4 \pi\left(\sigma_{0}+\sigma_{i}\right) & =-\frac{2 Q}{\alpha \cos \frac{p}{2}} \cdot \frac{1}{\sqrt{\tan ^{2} \frac{p}{2}-\tan ^{2} \frac{\theta}{2}}} \\
& =-\frac{4 Q}{\alpha} \frac{d}{d \theta} \sin ^{-1}\left(\frac{\sin \frac{\theta}{2}}{\sin \frac{p}{2}}\right)
\end{aligned}
$$

It is interesting to note that the total density $\sigma$ given by equation (18) is precisely that which would exist on the charged arc with a total charge $-Q$ in the absence of any external charges. The potential for this case may be found from equation (7) by subtracting from its second member the term $2 Q \log \frac{1}{z}$. Similarly adding to this side of the equations any term of the type $c \log z$ gives the corresponding potential when the line charge at the origin and the total charge on the conducting arc are each arbitrary. In the absence of any external charge, the densities would be given by interchanging the subscripts $_{i}$ and $\mathrm{o}$ in equations (16) and (17).

If we make the final transformation $z=Z^{\mathrm{n}}$ and let $\alpha=a^{\mathrm{n}}$ and $q=\frac{p}{n}$ where $a>0$, and $n$ is a positive integer, then the entire $z$ plane is repeated in each of the $n$ sectors of the $Z$ plane which is bounded by two straight lines radiating from the origin having an angular aperture $\frac{2 \pi}{n}$. Equation (7) then becomes

$$
\begin{aligned}
W= & Q \log \frac{a^{\mathrm{n}}-Z^{\mathrm{n}} \cos n q-\sqrt{\left(Z^{\mathrm{n}}-a^{\mathrm{n}} e^{\mathrm{Inq}}\right)\left(Z^{\mathrm{n}}-a^{\mathrm{n}} e^{-\mathrm{ln} q}\right)}}{4 \cos ^{2} \frac{n q}{2}} \\
& +Q \log \left[a^{\mathrm{n}} \cos n q-Z^{\mathrm{n}}-\sqrt{\left(Z^{\mathrm{n}}-a^{\mathrm{n}} e^{\mathrm{Inq}}\right)\left(Z^{\mathrm{n}}-a^{\mathrm{n}} e^{-\mathrm{ln} q}\right)}\right] \\
& -2 n Q \log Z-Q \log \sin ^{2} \frac{n q}{2}
\end{aligned}
$$


where the radical indicates that branch whose real part is negative when $|Z|<a$. Now

$$
z=r e^{\mathrm{i} \theta}=Z^{\mathrm{n}}=R^{\mathrm{n}} e^{\mathrm{in} \phi} \text { or }\left\{\begin{array}{l}
r=R^{\mathrm{n}} \\
\theta=n \phi
\end{array}\right.
$$

Hence, if the point $z$ describes the circular arc of radius $\alpha$ from $\theta=-p$ to $\theta=p$, the point $Z$ will describe the circular arc of radius $a$ from $\phi=-\frac{p}{n}=-q$ to $\phi=\frac{p}{n}=q$. Hence, there are $n$ equal, equally spaced, arcs of the circle $R=a$, each of angular width $2 q$, upon which the real part of the second member of equation (19) vanishes. When $\frac{z}{\alpha}$ is replaced by $\frac{Z^{\mathrm{n}}}{a^{\mathrm{n}}}=\frac{R^{\mathrm{n}}}{a^{\mathrm{n}}} e^{\mathrm{in} \phi}$ the expansion equation (10) gives

$$
U=2 n Q\left\{\log \frac{a}{R\left(\sin \frac{n q}{2}\right)^{n}}-\cos ^{2} \frac{n q}{2}\left(\frac{R}{a}\right)^{n} \frac{\cos n \phi}{n} \ldots\right\}
$$

If $\frac{R}{a}$ is as small as $\frac{1}{10}$ and $n$ as large as 8 , the second term of this expression is of the order of $10^{-9}$ and may certainly be neglected. The potential $U$ is then a function of $R$ only. Over the circle $R=b$ where $\left(\frac{b}{a}\right)^{\mathrm{n}}$ is small, the potential $U$ has the constant value $U_{\mathrm{b}}$ where ${ }^{2}$

$$
U_{\mathrm{b}}=Q \log \frac{a^{2 \mathrm{n}}}{b^{2 \mathrm{n}} \sin ^{2} \frac{n q}{2}}
$$

This equation enables one to eliminate the charge $Q$ from the equation for the potential, introducing instead the potential $U_{\mathrm{b}}$ of the inner rod. For the purpose of computing $U$ or drawing lines of force, it will probably be more convenient to do this first for the $z$ plane using the polar coordinates $r$ and $\theta$, by means of the equation (9). The corresponding points in the $Z$ plane are then found by replacing $r$ by $R^{\mathrm{n}}, \theta$ by $n \phi$, and $\alpha$ by $a^{\mathrm{n}}$.

The computation of the potential $U$ for any point $(r, \theta)$ in the plane may best be made by evaluating the four radii $r_{1}, r_{2}, r_{3}$, and $r_{4}$, and the

2 It may be noted that the electric field intensity under these circumstances is very uniform at all points of the inner cylinder of radius $b$, its value being

$$
-\left(\frac{\partial U}{\partial R}\right)_{\mathrm{R}=\mathrm{b}}=\frac{U_{\mathrm{b}}}{b \log \frac{a}{b}} \cdot \frac{1}{1+\frac{\log \csc \frac{n q}{2}}{n \log \frac{a}{b}}}
$$

This reduces to the known value when the circular arcs touch, thus closing the circle, for in that case

$$
\frac{n q}{2}=\frac{\pi}{2} \text { so that } \log \csc \frac{n q}{2}=0
$$


angles $\theta_{1}, \theta_{2}, \theta_{3}$, and $\theta_{4}$ in terms of $r$ and $\theta$ according to their definitions by the equation (8). This may be done either graphically or arithmetically ( $p=n q$ and $\alpha=a^{\mathrm{n}}$ must be given). The explicit formula for the potential $U$ at any point in the plane is then found by taking the real part of equation (9). It is

where

$$
U\left(r_{1} \theta\right)=\frac{Q}{2} \log \frac{f\left(r_{1}, r_{2}, \cdots r_{4}, \theta_{1} \cdots \theta_{4}\right)}{f\left(-r_{1}, r_{2} \cdots r_{4}, \theta_{1} \cdots \theta_{4}\right)}
$$

$$
\begin{aligned}
f\left(r_{1}, r_{2} \cdots r_{4},\right. & \left.\theta_{1} \cdots \theta_{4}\right)= \\
& =r_{1}^{2} \cos ^{2} \frac{p}{2}+r_{3}^{2} \sin ^{2} \frac{p}{2}+r_{2} r_{4} \\
& +2 r_{1} r_{3} \sin ^{2} \frac{p}{2} \cos ^{2} \frac{p}{2} \cos \left(\theta_{3}-\theta_{1}\right) \\
& +2 r_{1} \sqrt{r_{2} r_{4}} \cos ^{2} \frac{p}{2} \cos \left(\frac{\theta_{2}+\theta_{4}}{2}-\theta_{1}\right) \\
& +2 r_{3} \sqrt{r_{2} r_{4}} \sin ^{2} \frac{p}{2} \cos \left(\frac{\theta_{2}+\theta_{4}}{2}-\theta_{3}\right)
\end{aligned}
$$

Washington, March 20, 1926. 